\title{
APORTES SEDIMENTARIOS DE LOS RÍOS LLUTA Y SAN JOSÉ EN LA ZONA COSTERA DE LA RADA DE ARICA, CHILE
}

\author{
LLUTA AND SAN JOSÉ RIVERS SEDIMENTARY FLUVIAL CONTRIBUTIONS \\ TO THE COASTAL AREA IN ARICA HARBOUR, CHILE.
}

\author{
Humberto Campos Ortega ; Gonzalo Díaz Muñoz ${ }^{2}$; Claudio Campos Ortega ${ }^{3}$
}

\begin{abstract}
RESUMEN
El cauce del río San José, en el norte de Chile, atraviesa la ciudad de Arica, aporta suministro hídrico en la parte alta y media del valle de Azapa, como resultado de aportes pluviales. Este río nace en la cordillera Central o límite poniente del Altiplano Andino (JICA, 1995). Sus cursos superiores son cauces estrechos, encajonados y sinuosos, limitando el desarrollo del suelo y la agricultura. En su curso medio, el cauce se ensancha. El río San José tiene un caudal permanente, pero en cantidades reducidas sólo en su curso superior, recurso que se puede utilizar localmente para desarrollar una agricultura de subsistencia. Sólo en los meses estivales del invierno altiplánico -entre diciembre y marzo- en la forma de torrente aluvional logra alcanzar su curso inferior e incluso al mar, razón por la cual se ajusta a la definición de río efímero planteada por Wetzel (1975). En los meses de excedentes hídricos en la precordillera se producen infiltraciones que recargan las napas subterráneas y/o alimentan las vertientes existentes en las secciones inferiores del cauce de descarga. Por su parte, el río Lluta, ubicada su parte baja unos $10 \mathrm{~km}$ al norte de Arica, presenta escurrimiento exorreico (Goldman \& Horne, 1994; Wetzel, 1975) permanente en sentido este-oeste y su cuenca se clasifica como preandina, la cual tiene una longitud de $147 \mathrm{~km}$ y son sus principales tributarios el río Azufre y las Quebradas de Caracarani, Colpitas y Socoroma. Como la mayoría de las cuencas principales de la región, ésta se caracteriza por la escasez o baja frecuencia de precipitaciones estacionales. El río Lluta, en el acuífero superficial, se caracteriza por altos contenidos de boro (B) (Aquaconsult Cía. Ltda., 1998). La concentración de B en el río Lluta oscila entre 16 y 25 mg/L, por lo que clasifica en agua clase 4 de acuerdo con la Norma de Calidad $2^{\mathrm{a}}$ en Aguas Marinas. En términos generales, la ocurrencia de aluviones en ambas cuencas, como eventos torrenciales de cierta consideración tanto en volumen como en efectos, produce la erosión del cauce, terrenos adyacentes y suelos agrícolas aledaños, provocando la pérdida de suelos productivos y sus cultivos, además de daños en bocatomas y sistemas de riego. Estos aluvionales aperiódicos destruyen también caminos, vías férreas y redes de agua potable, impactando la ciudad y otros asentamientos humanos rurales; contribuyen al embancamiento del puerto comercial, deteriorando playas del litoral marino por el transporte de material particulado suspendido y sedimentable hacia ese sector costero. El objetivo de este trabajo es cuantificar el material particulado suspendido y sedimentable (STSS) de origen continental transportado fluvialmente al sector costero de la rada de Arica durante eventos aluvionales estivales. La metodología de muestreo y análisis de STSS siguió los protocolos indicados en APHA, AWWA, WPCF. MÉTODOS NORMALIZADOS para el Análisis de Aguas Potables y Residuales. Ediciones Díaz de Santos, S. A. (1992). Los resultados coinciden con lo observado hace algunos años, cuando uno de los muelles del sector Chinchorro quedó embancado. El registro de ST en la desembocadura del Lluta en febrero de 2005 fue de 1.705,47 ton/día, mientras que el San José aportó 74,22 ton/día, durante uno de los días de mayor caudal. El material particulado fluvial afecta notablemente el turismo de la ciudad, alejando a residentes y turistas de playas del sector norte. Estos aportes contribuyen al embancamiento de la dársena del puerto comercial. El río Lluta hace mayor aporte estacional de material sedimentario que el río San José, pero este último produce mayor impacto en infraestructura pública y turismo.
\end{abstract}

Palabras clave: Cauce, aluvional, particulado, sedimentable, fuerza iónica.

\footnotetext{
1 Depto. de Química. Facultad de Ciencias. Univ. de Tarapacá. camposortegahumberto@gmail.com

2 Cesmec Chile.goyls@hotmail.com

3 Depto. Química y Biología. Univ. de Atacama; ccamposortega@ gmail.com
}

Fecha de Recepción: 16 Mayo 2006

Fecha de Aceptación: 03 Julio 2006 


\begin{abstract}
San José river bed in the north of Chile runs across Arica city providing water supplies in the middle and high lands of Azapa valley as a result of pluvial contributions. This river rises in the central mountain range or west border of the Andean High Plateau (JICA,1995). Its superior courses are narrow, incised and sinuous ditches which prevent soil and agriculture development.The ditch becomes wider in its middle course. San José River has a permanent flow but with small quantities only in its upper course, resource which can be used locally in order to develop subsistence farming agriculture. It is only in the summer months of the High Plateau winter, between December and March, that the river as a flood stream reaches its lower course and also the ocean.That is why it has been defined by Wetzel (1975) as ephimeral river. During the months of water surplus in the Andean Plateau occur some infiltrations which recharge the water tables and/or supply water springs in the inferior course of the river. On the other hand, the lower course of Lluta river at 10 kilometers north of Arica shows permanent east-west exoreic draining (Goldman \& Home, 1994; Wetzel, 1975) and its basin of $174 \mathrm{kms}$ long is classified as Pre-Andean, being its main tributaries Azufre river and Caracarani, Colpita and Socoroma gorges. As many of the main basins in the region, this one shows little or no seasonal rains. The Lluta river is characterized by its high borum (B) concentrations (Acquaconsult Cía Ltd. 1998). The B concentration in Lluta river is between 16 and $25 \mathrm{mg} / \mathrm{L}$, being labelled as class 4 water according to the Secondary Quality Norm in Sea Water. In general, alluvia in both basins as torrential events of relative magnitude in volume and effect produce erosion in both beds, adjacent lands, and farming soil which in turn causes damage to crops, productive soil, and irrigation systems.These non-periodic alluvia also destroy roads, railways, tap water systems, damaging Arica city and other rural human settlements; they also contribute to block the commercial port, spoiling beaches in the shoreline with settling and suspended particulate matters (STSS) of continental origin. The purpose of this research is to quantify the STSS which is carried to the shoreline of Arica's harbour during summer alluvial events. The sampling methodology and STSS analysis followed the protocols indicated in APHA,AWWA,WPCF. NORM METHODS for tap and residual waters analysis. Díaz de Santos Ed., S.A. (1992). Results coincide with what it was observed some years ago when one of the docks in the Chinchorro area became blocked. The ST report at the Lluta river mouth in February 2005 was 1.705,47 t/day whereas San José river showed a 72.22 t/day during the highest flow days. The fluvial particulate matter greatly damages the city tourism preventing residents and tourist to visit the noth area beaches. These sediments cause the seaport dock become blocked. The Lluta river provides more seasonal sediments than San Jose river, nevertheless the latter causes more damage in public infrastructure and tourism.
\end{abstract}

Key words: Bed, alluvial, particled, sedimentable, ionic power.

\section{INTRODUCCIÓN}

El ser humano, especie animal está inserta en un espacio ambiental propio como es la antrósfera, mientras que la generalidad de las especies vivas se ubica en la biosfera. El humano ha utilizado de manera exitosa los recursos naturales del entorno, asegurando su permanencia en el tiempo. Los recursos naturales están disponibles en diferentes espacios ambientales, entre los cuales se puede considerar una cuenca hidrográfica, unidad geomorfológica que la especie humana comparte con otros seres vivos, desarrollándose una relación biocenótica dinámica y armónica entre las especies de los biotopos.

La cuenca hidrográfica es una porción de la superficie terrestre cuyas aguas fluyen hacia cauces de ríos, lagos o la zona costera del mar. La cuenca está delimitada por la divisoria de aguas de las altas cumbres que la abastecen, generando de esta forma un sistema dinámico de cauces superficiales y/o subterráneos de diversas magnitudes, que al confluir en un cauce único (o río) colectan el aporte volumétrico de las aguas lluvia que dentro de dicha cuenca se producen en horas, días o años. Esta área territorial se considera en la actualidad la unidad natural "generadora" de recursos disponibles, sean éstos biológicos, abióticos, sociales y económicos requeridos por el ser humano para su desarrollo. Por lo tanto, la concepción moderna de planificación y manejo del territorio centran su objetivo en la administración racional de los recursos naturales contenidos en cada cuenca, siendo el hilo conductor de esta preocupación el recurso hídrico disponible dentro de sus límites.

El aporte hídrico proviene de las precipitaciones estacionales que en toda cuenca se producen, las cuales, resultado de la disponibilidad y ocurrencia de las mismas, definen el comportamiento del aporte en la cabecera o parte alta del área, el efecto de transporte en la sección media o de escurrimiento y, en la parte baja, los fenómenos de sedimentación o decantación en las zonas lentas del río o en su descarga. El escurrimiento del recurso hídrico superficial y/o subterráneo por la cuenca es la fuente del agua requerida por la vida en sus diversas manifestaciones, para satisfacer requerimientos hídricos de organismos simples hasta las necesarias para el actual modelo de desarrollo social y económico de la antrósfera, es decir, sus componentes hidrológicos, ecológicos, ambientales y socioeconómicos. 
Como concepto ambiental se puede reconocer en toda cuenca la importancia reguladora de la recarga hídrica y de los ciclos biogeoquímicos, permitiendo la conservación de la biodiversidad que mediante sus procesos biológicos y fotoquímicos ayuda a fijar el $\mathrm{CO}_{2}$ contribuyendo con procesos de edafogénesis en los suelos locales. Estos procesos permiten potenciar la diversidad de etapas o procesos naturales requeridos para asegurar la calidad física y química del agua disponible, componente ambiental fundamental para asegurar la adecuada expresión de vegetales y animales en cualquier ecosistema.

La captación natural de las diferentes formas de precipitaciones - pluvial o nival- le permite a la cuenca generar un volumen de aguas disponibles, las cuales son almacenadas de forma natural en diversas formas y tiempos de disponibilidad, hasta que son liberadas en forma de evaporación o aguas de escurrimientos subterráneos o superficiales, en forma de cauces regulares o eventos torrenciales aluvionales. Estas son las fuentes hídricas requeridas por la antrósfera como fuente de suministro de recursos naturales para su sustento, los que mediante procesos de agregación de valor generan las herramientas necesarias para desarrollar social y culturalmente la sociedad.

\section{ANTECEDENTES GENERALES}

De acuerdo con informes de propuestas presentadas al Ministerio de Obras Públicas (MOP), la cuenca del valle de Azapa o del río San José se ubica administrativamente en las provincias de Arica y Parinacota, Región de Tarapacá, y es ella importante a nivel regional desde el punto de vista del desarrollo agropecuario, pues dispone para agricultura actualmente de unas 2.620 ha bajo canal, en las cuales se explotan importantes rubros tales como olivos, tomates, ajíes y frutos tropicales.

El suministro hídrico del valle de Azapa proviene del río San José como resultado de aportes pluviales, río que nace en la cordillera Central o límite poniente del Altiplano Andino. Los cursos superiores de este río se caracterizan por sus cauces estrechos, encajonados y sinuosos, que permiten un limitado desarrollo del suelo y la agricultura. En su curso medio y una vez pasada la sierra de Huaylillas, el cauce se ensancha permitiendo un adecuado proceso de edafogénesis que permite generar suelos aptos para uso agropecuario, los cuales presentan limitaciones por el contenido salino menor al del río Lluta (Campos, 2005).

El río San José ostenta un caudal permanente, pero en cantidades reducidas sólo en su curso superior, recurso que se puede utilizar localmente para desarrollar una agricultura de subsistencia. Sólo en los meses estivales del invierno altiplánico -entre diciembre y marzo- su caudal en la forma de torrente aluvional logra alcanzar a su curso inferior e incluso al mar, razón por la cual se ajusta a la definición de río efímero planteada por Wetzel (1975). En los meses de excedentes hídricos en la precordillera se producen infiltraciones que recargan las napas subterráneas y/o alimentan las vertientes existentes en las secciones inferiores del cauce de descarga.

El desarrollo agrícola actual del valle de Azapa está fuertemente limitado por la cantidad y calidad de los recursos hídricos existentes. Estudios recientes de la Dirección de Obras Hidráulicas (D.O.H.) asignan a este valle una superficie agrícola potencial de 4.450 ha, área de explotación superior en un $70 \%$ a los terrenos actualmente cultivados bajo canal (2.620 ha).

Más al norte de la hoya antes descrita se ubica el valle de Lluta con su río, el cual corresponde a una de las principales cuencas hidrográficas de la Región de Tarapacá, la que se extiende entre los paralelos $18^{\circ}-18^{\circ} 30^{\prime}$ latitud sur (S) y meridianos $70^{\circ} 20^{\prime}-69^{\circ} 22^{\prime}$ longitud oeste (W). Abarca una superficie de $3.378 \mathrm{~km}^{2}$. Cubre parcialmente el territorio de las provincias de Parinacota y Arica, siendo la localidad de Putre el poblado más importante (D.G.A. 2004).

Como se indica en el Diagnóstico y Clasificación de los Cursos y Cuerpos de Agua Según Objetivos de Calidad para la cuenca del río Lluta, trabajo solicitado el año 2004 por la Dirección General de Aguas (D. G. A.), este río presenta escurrimiento exorreico (Goldman \& Horne, 1994; Wetzel, 1975) permanente en sentido este-oeste y su cuenca se clasifica como preandina, la cual tiene una longitud de $147 \mathrm{~km}$ y son sus principales tributarios el río Azufre y las Quebradas de Caracarani, Colpitas y Socoroma. Como la mayoría de las cuencas principales de la región, ésta se caracteriza por la escasez o baja frecuencia de precipitaciones estacionales, fenómeno que explica la escasa vegetación tanto en diversidad como en cantidad presente en sus suelos, exceptuándose el sector bajo del valle, el 
cual es un área donde estos suelos tienen un uso predominantemente agrícola.

Por la importancia que representa la desembocadura del Lluta para la avifauna local, esta área se incorporó bajo la protección oficial del Sistema Nacional de Áreas Silvestres Protegidas por el Estado (SNASPE), pues es éste el más importante de los humedales costeros de la I Región. Desde el punto de vista de la biodiversidad constituye este sitio un hábitat fundamental para la avifauna acuática local y/o migratoria, pues en el lugar se presenta una interesante diversidad de especies, la que ha generado preocupación en la municipalidad de Arica, la cual ha solicitado parte de los terrenos que comprenden la desembocadura para crear un Parque Natural Municipal, intentando proteger el área de la activa intromisión de residentes y turistas en los ciclos de postura e incubación de nuevas generaciones de aves.

Este cauce recibe descargas de tipo agrícola derivadas de actividades de riego en su parte alta (Putre y Socoroma), mientras que en el valle de Lluta o sección media los recibe desde Chapisca y pasando por Poconchile hasta la parte baja del valle, en cuyo cauce se descargan además residuos industriales líquidos (riles), como los efluentes salinos de las aguas de descarte provenientes de la planta desalinizadora de la empresa Desalari, los que ingresan al río en su desembocadura (Figura 1).

El río Lluta se caracteriza por altos contenidos de boro, presentándose el elemento como una tendencia creciente a lo largo del curso del río, efecto que se atribuye a depósitos salinos como boratos, abundantes en el norte de Chile. La concentración de este elemento presente en el río Lluta oscila entre 16 y $25 \mathrm{mg} / \mathrm{L}$, por lo que clasifica en agua

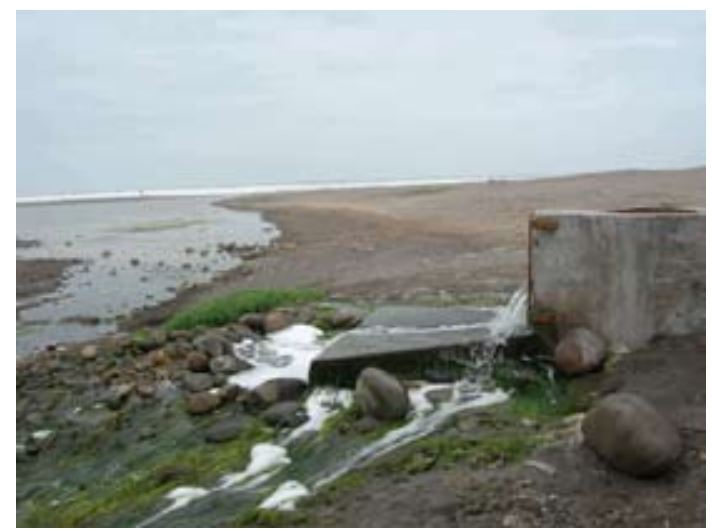

Figura 1. Descarga Agua Descarte Desalari. clase 4 de acuerdo con la Norma de Calidad $2^{\mathrm{a}}$ en Aguas Marinas.

Como lo demuestran diversos trabajos científicos, el río Lluta y sus tributarios principales presentan serias limitaciones como agua para fines de riego (NCh $\mathrm{N}^{\mathrm{o}} 1333$ Of. 84), resultado de un activo proceso de contaminación natural. Dicha limitante, expresada como Sólidos Totales Disueltos (STD) se manifiesta en las características concentraciones de especies minerales disueltas en estas aguas, destacando entre ellas elevadas concentraciones de B, As, compuestos inorgánicos complejos y iones metálicos, parámetros activados por la singular dinámica climática de zonas áridas. Este proceso climático, caracterizado por las escasas precipitaciones y una alta evaporación en la cuenca, dinamiza el proceso de formación de salares en las planicies altiplánicas y de la edafogénesis de suelos agrícolas salinos en sus secciones medias, pues la falta de aportes de aguas lluvia no permite formar suelos lavados ni diluir los contaminantes presentes en el río, los que se van así concentrando en su desplazamiento por la cuenca al fluir en dirección al mar.

Como indican otros informes, en términos generales la ocurrencia de aluviones en ambas cuencas como eventos torrenciales de cierta consideración, tanto en volumen como en efectos, producen la erosión del cauce, terrenos adyacentes y suelos agrícolas aledaños, provocando la pérdida de suelos productivos y sus cultivos, además de daños en bocatomas y sistemas de riego. Estas pérdidas en la agricultura no son únicas ni las más importantes de las provincias de Arica y Parinacota, ya que estos aluviones destruyen también infraestructura pública (caminos, vías férreas y redes de agua potable), producen daños en la ciudad y en otros asentamientos humanos rurales; son los responsables del embancamiento del puerto comercial, del deterioro de playas y de su entorno litoral como consecuencia del arrastre de material particulado sedimentable hacia el ambiente acuático marino. Este material se afecta resultado de la fuerza iónica que presenta el cuerpo oceánico sobre el material sedimentable arrastrado, fuerza que estimula la sedimentación, favoreciendo el embancamiento de la costa con material sedimentado, fenómeno observado hace algunos años en playas del sector Chinchorro, cuando uno de los muelles quedó prácticamente en terreno seco. Además, el material particulado suspendido repercute fuertemente en la empresa del turismo de Arica, al alejar a residentes y turistas 
de playas o sectores costeros de recreación estival. El efecto de estos aportes se duplica en la rada del puerto comercial, resultado de los aportes de los ríos Lluta y San José, de Arica.

Durante el invierno altiplánico del año 2001 las crecidas de los ríos Lluta y San José ocasionaron daños valorizados preliminarmente en varios millones de dólares (Referencia: Ministerio de Obras Públicas). De los efectos antes descritos se observa el traslado recurrente o erosión de suelos agrícolas, los que se transforman en sedimentos marinos costeros con el consiguiente deterioro por embancamiento observado en sitios de operaciones portuarias, fenómeno favorecido por el área de abrigo que produce el molo, el cual al disminuir la intensidad del oleaje y velocidad de la corriente permite mayor tiempo de sedimentación, obligando a frecuentes dragados en los sitios de atraque de la dársena portuaria. También se ha observado la aparición de nuevas estructuras litorales, como la península generada por aluviones en la ribera norte de la desembocadura del río San José desde aproximadamente el verano de 1973.

La situación agropecuaria descrita y los daños reseñados han conducido a todos los sectores regionales a solicitar una solución de carácter urgente. Es por ello que se han discutido, analizado y gestionado diversos proyectos relativos a la construcción de embalses con estudios en las cuencas de los ríos San José y Lluta. Estas obras regulatorias de riego han sido propuestas como una forma de controlar y regular la bajada de los ríos, buscando principalmente mitigar así el impacto económico y social que producen los desbordes descontrolados de ambos ríos. Para el diseño de estas obras sería conveniente considerar los resultados aportados en este trabajo.

El río Lluta, cuya cuenca hidrográfica se distribuye por las provincias de Arica y Parinacota, Región de Tarapacá, también es importante desde el punto de vista del desarrollo agropecuario de Arica, pues su repercusión económica es regional derivada del volumen de producción agrícola. En la desembocadura de este río no se observa el embancamiento de la zona costera, resultado de la exposición del materia suspendido a un tren de olas de mayor persistencia y a ondas de marea, condiciones que permiten un mayor tiempo de residencia o suspensión del material particulado terrígeno transportado por el arrastre fluvial o descarga en el sector norte de la rada de Arica. Esta mayor energía cinética de las corrientes marinas dispersa el material particulado suspendido y sedimentable costa afuera, el que al ser comprimido por la fuerza iónica del agua de mar sedimentará principalmente cuando la dilución marina alcance alrededor del $10 \%$ o de salinidad que corresponde al filtro geológico de un ambiente acuático transicional, contribuyendo así a la someridad de la rada de Arica. Aun cuando no se observan alteraciones costeras de importancia derivadas de aluviones del Lluta, se presume el traslado de una cantidad no menor de suelos agrícolas erosionados derivado del desgaste producido por la fuerza del agua en el cauce, particularmente en aquellas zonas erosionables del río y en los terrenos agrícolas adyacentes (Goldman \& Horne, 1994).

\section{ÁREA DE ESTUDIO}

Ambos cauces se presentan en la foto satelital de la Figura 2, en la cual se indican las estaciones permanentemente utilizadas para colectar muestras de aguas en cada río, durante los eventos aluvionales descritos.

Tabla 1

Identificación de Estaciones en estudio

\begin{tabular}{|l|c|}
\hline ESTACIÓN & $\mathbf{N}^{\mathbf{0}}$ Estación \\
\hline Azapa, Las Maitas & E - 1 \\
\hline Azapa, Rotonda & E - 2 \\
\hline Azapa, Puente Ferrocarril & E - 3 \\
\hline Azapa, Desembocadura & E - 4 \\
\hline Lluta, Poconchile & E - 5 \\
\hline Lluta, Chacabuco & E - 6 \\
\hline Lluta, Sta. Lucía & E - 7 \\
\hline Lluta, Panamericana & E - 8 \\
\hline Lluta, Desembocadura & E - 9 \\
\hline
\end{tabular}

Las características de cada una de las estaciones indicadas en la Tabla 1 se observan en las siguientes fotos, la mayoría de las cuales se tomaron durante el período aluvional.

\section{MATERIALES Y MÉTODOS}

Estaciones de Muestreo: Fueron seleccionadas en la sección baja de ambos cauces por ser ésta la zona de mayor concentración de material 


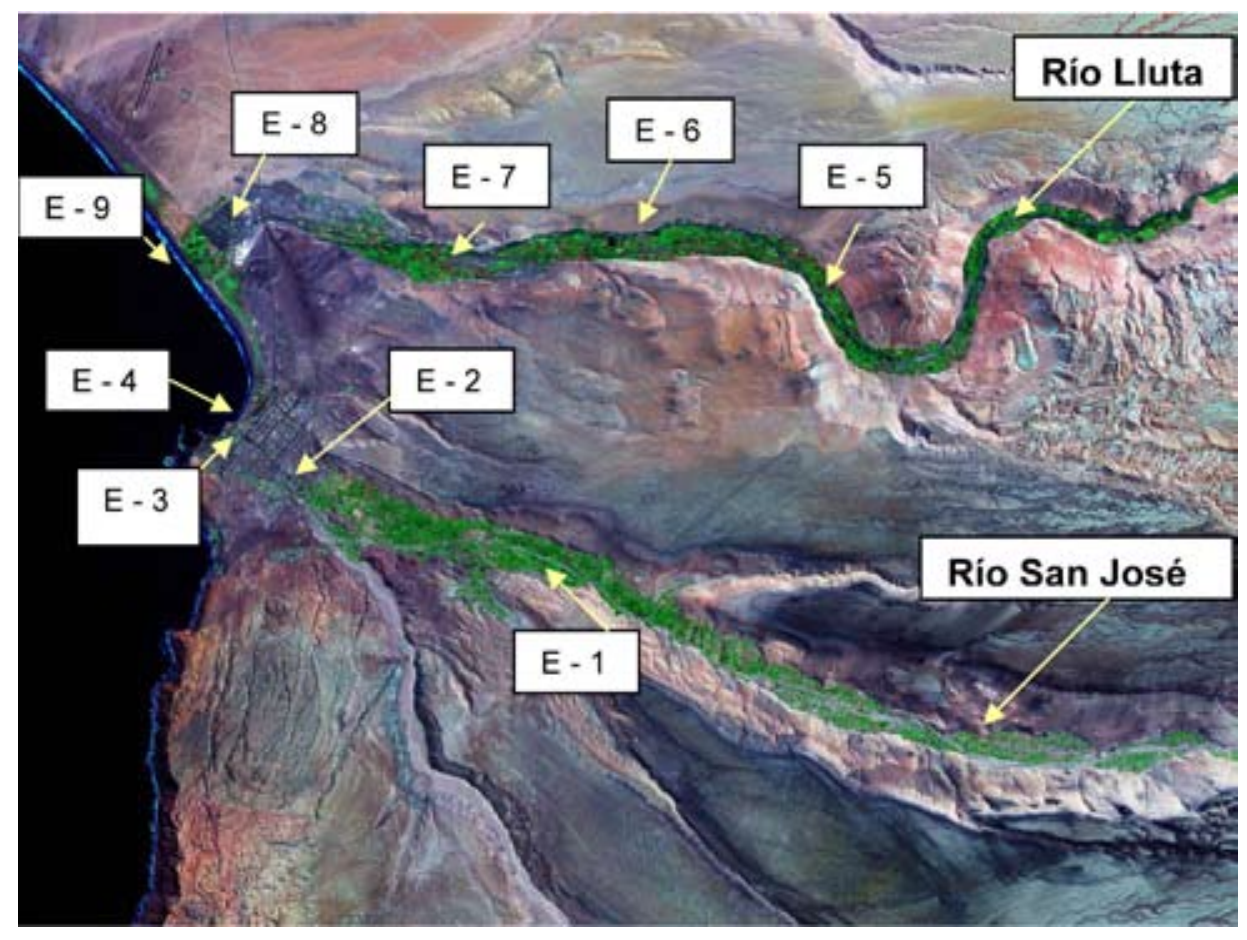

Figura 2. Estaciones de muestreo en los ríos del estudio. Fuente: Landsat 2000. NASA.

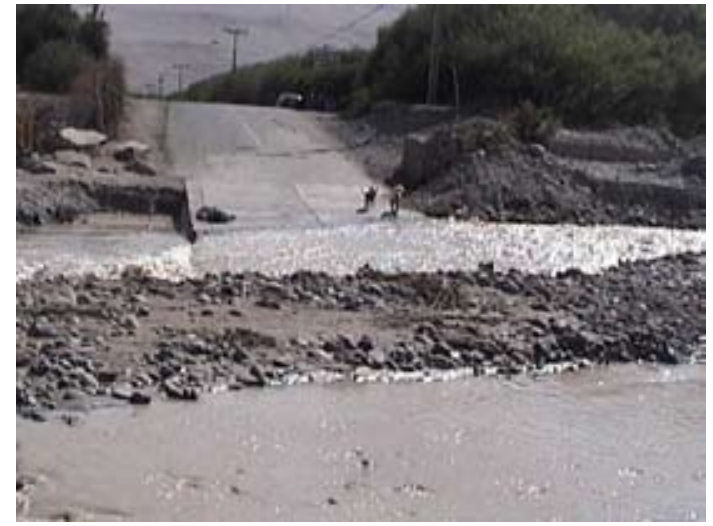

Figura 3. Estación $\mathrm{N}^{\circ} 1$ de Muestreo. Sector Las Maitas. Azapa.

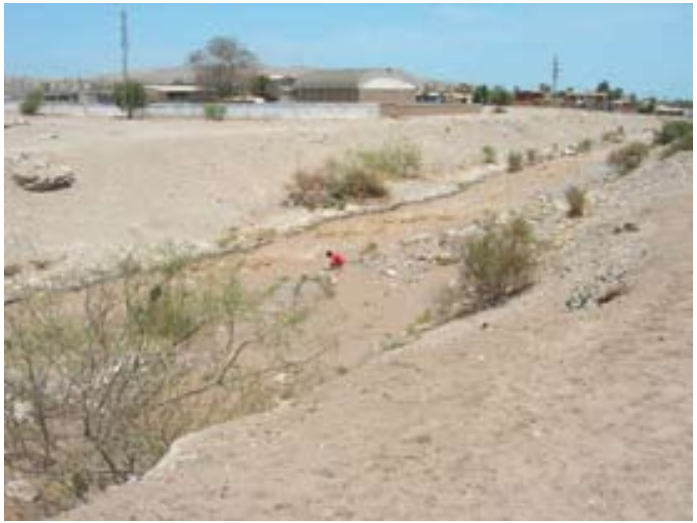

Figura 4. Estación $N^{o} 2$ de Muestreo. Sector Rotonda. Azapa. 


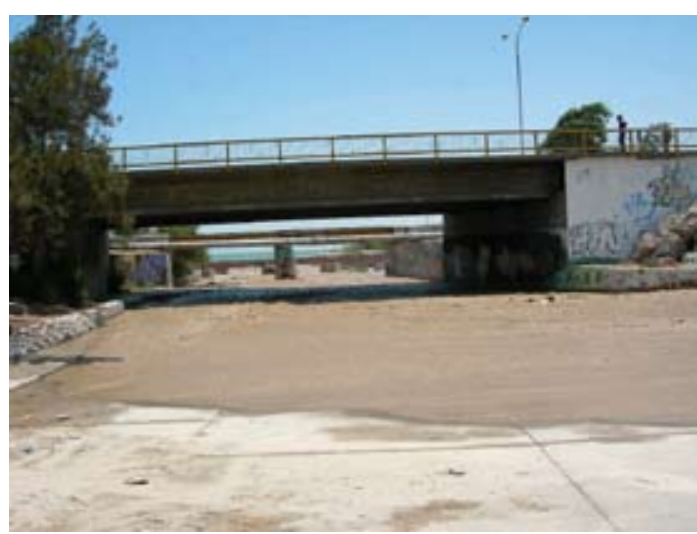

Figura 5. Estación $N^{\circ} 3$ de Muestreo. Sector Puente Ferroviario. río San José.

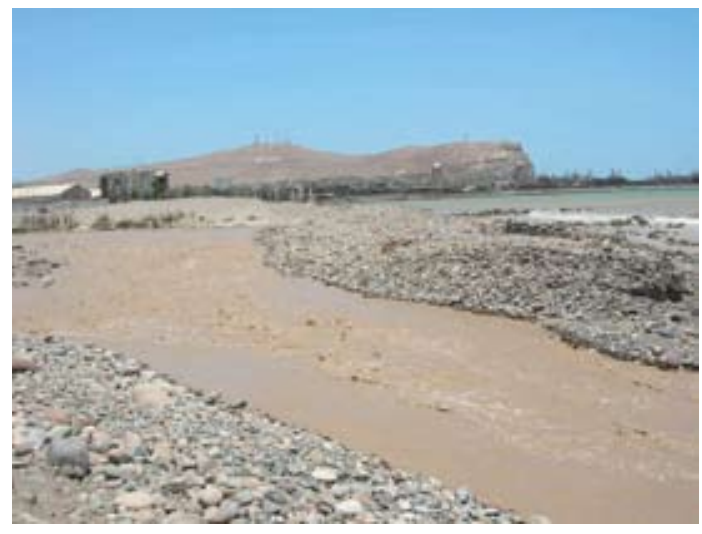

Figura 7. Estación $N^{\circ} 4$ de Muestreo. Sector Desembocadura río San José.

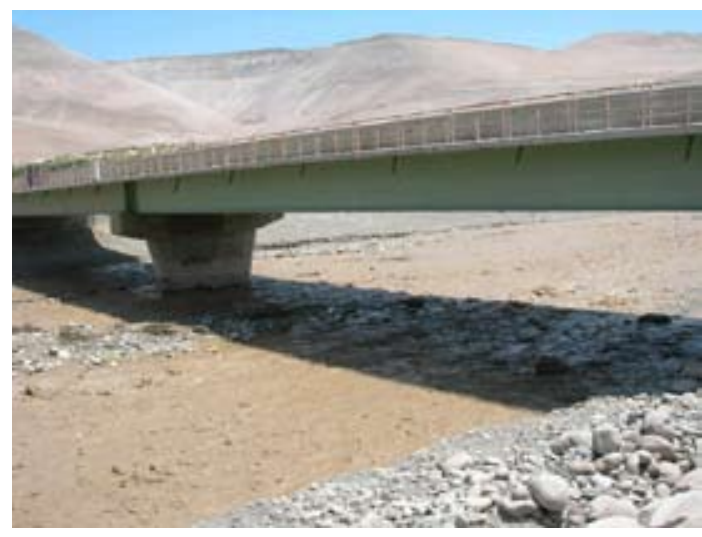

Figura 9. Estación No 6 de Muestreo. Sector Puente Santa Lucía, río Lluta.

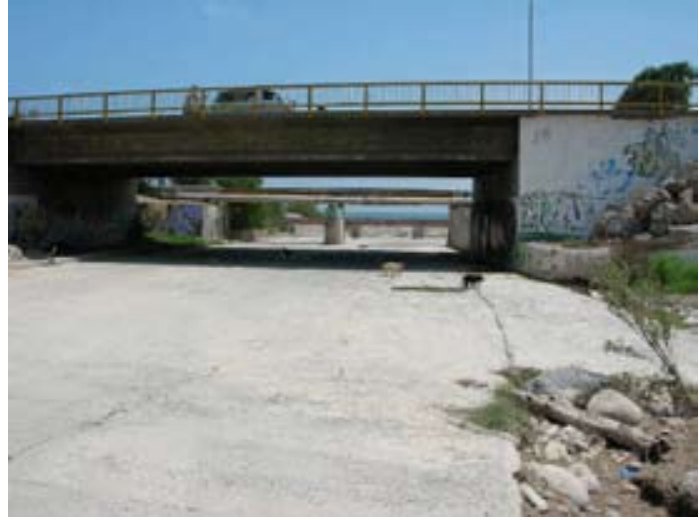

Figura 6. Sector Puente Ferroviario: río San José, sin bajada del río.

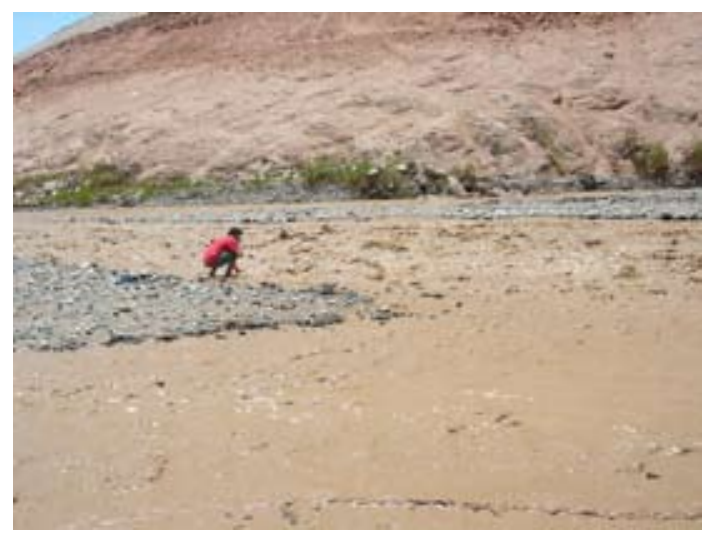

Figura 8. Estación $N^{\circ} 5$ de Muestreo. Sector Poconchile, río Lluta.

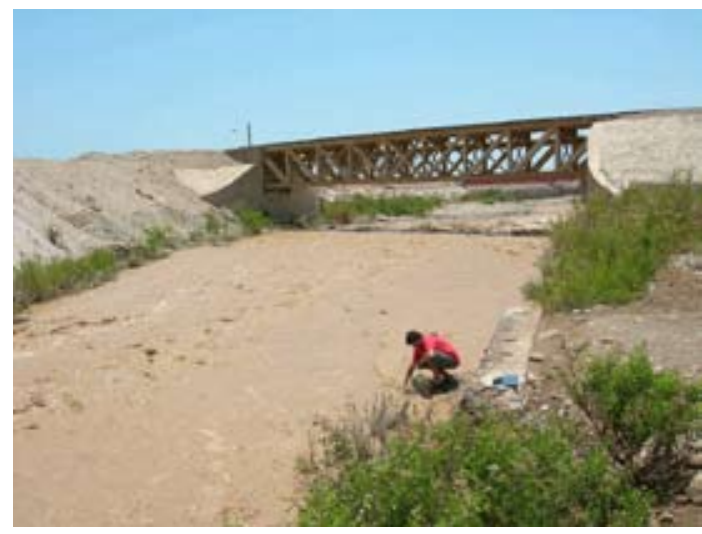

Figura 10. Estación No 7 de Muestreo. Sector Puente Ferroviario, río Lluta. 


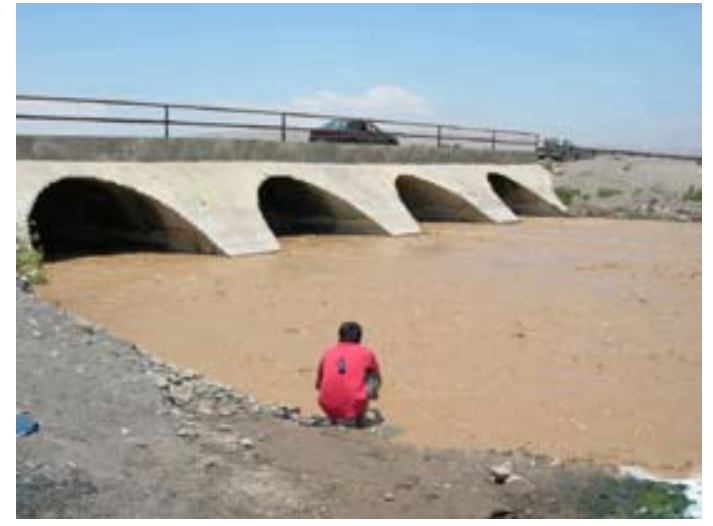

Figura 11. Estación $\mathrm{N}^{\circ} 8$ de Muestreo. Sector Puente Desembocadura, río Lluta.

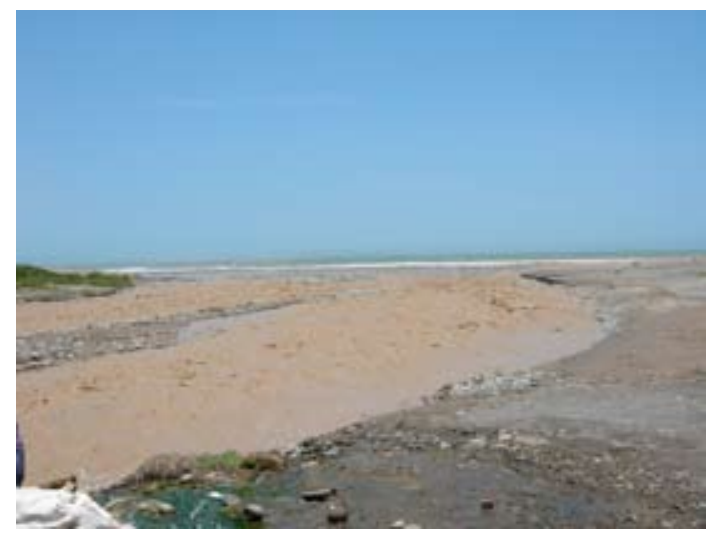

Figura 13. Estación $N^{\circ} 9$ de Muestreo. Sector Desembocadura, río Lluta.

sedimentable y también por su cercanía a la ciudad, donde se observan con mayor intensidad los efectos de esta sedimentación sobre el borde costero e infraestructura pública.

De acuerdo con la Tabla 1, en Azapa se establecieron estaciones en Las Maitas (E-1), Rotonda Saucache (E-2), Puente Ferrocarril (E-3) y Desembocadura (E-4). En Lluta, las estaciones corresponden a Poconchile (E- 5), Puente Chacabuco (E-6), Puente Santa Lucía (E-7), Puente Panamericana (E-8) y Desembocadura (E - 9).

Muestreo: Se registran datos obtenidos en épocas estivales de los años 1997, 2000, 2001, 2004 y 2005, como se indica en la fecha de colecta de muestras.

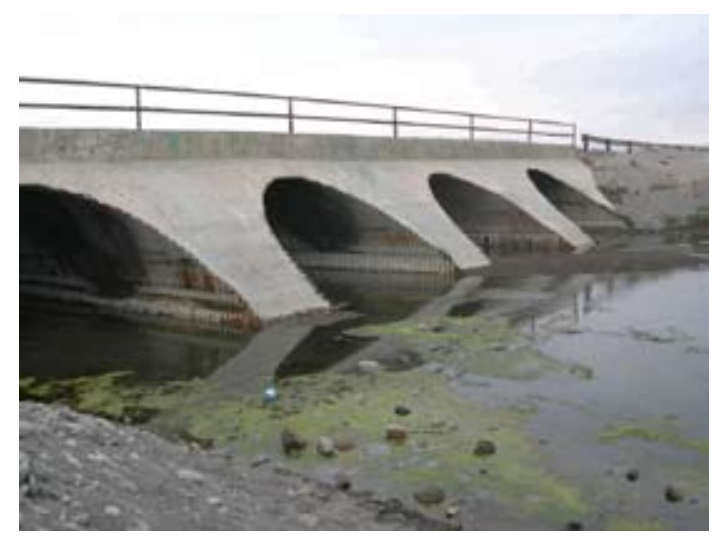

Figura 12. Sector Puente Desembocadura, río Lluta sin bajada del río.

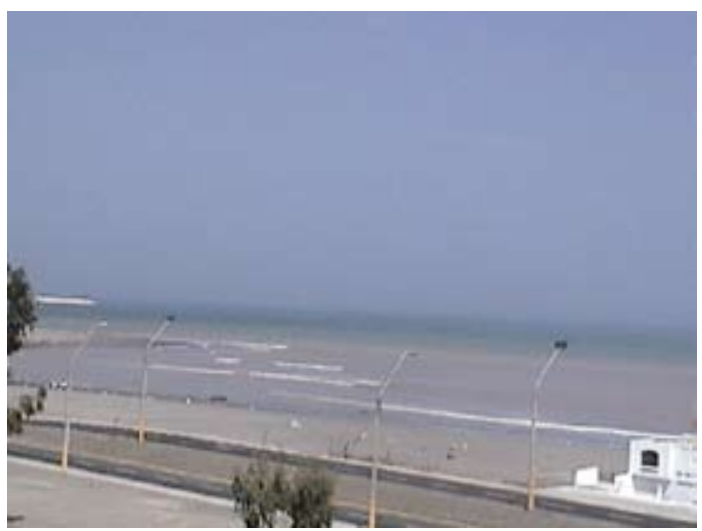

Figura 14. Aspecto del sector costero Chinchorro con evento aluvional.

Metodología de Análisis: Las muestras se colectaron y transportaron al laboratorio, siguiendo el protocolo indicado en la Norma Chilena $\mathrm{N}^{\circ}$ 411/6. Of. 98 (1998). En el laboratorio fueron procesadas aplicando filtración por $0,45 \mu \mathrm{m}$, en filtro de ester de celulosa, en muestras a determinar sólidos totales disueltos (STD) o solubles en el filtrado, según protocolo $2540 \mathrm{C}$, indicado en MÉTODOS NORMALIZADOS para el Análisis de Aguas Potables y Residuales (1992). Los sólidos suspendidos (SS) se midieron con el protocolo $2540 \mathrm{D}$, mientras que los sólidos totales (ST) se determinaron siguiendo el protocolo $\mathrm{N}^{\circ} 2540 \mathrm{~B}$, indicado en el ya mencionado apoyo bibliográfico. 


\section{RESULTADOS Y DISCUSIÓN}

RESULTADOS

Tabla 2

Caudales Promedio Azapa y Lluta

\begin{tabular}{|l|c|c|c|}
\hline Río & Año & Periodo & Q Promedio $\left(\mathbf{m}^{\mathbf{3}} / \mathbf{s e g}\right)$ \\
\hline San José (a) & 1997 & 4 a 7 de marzo & $8.020^{*}$ \\
\hline Lluta (c) & 1997 & 28 febr. a 3 de marzo & $37.950 *$ \\
\hline San José (a) & 2004 & 15 de febrero & $1.939 * *$ \\
\hline San José (a) & 2005 & 16 de febrero & $1.670 * *$ \\
\hline Lluta (b) & 2004 & 15 de febrero & $7.517 * *$ \\
\hline Lluta (c) & 2004 & 15 de febrero & $8.261 * *$ \\
\hline Lluta (b) & 2005 & 16 de febrero & $1.566 * *$ \\
\hline Lluta (c) & 2005 & 16 de febrero & $4.053 * *$ \\
\hline
\end{tabular}

a: Registro Ausipar. Azapa. b: Registro Alcérreca. Lluta. c: Registro Tocontasi. Lluta.

* Caudal Promedio río San José entre 4 y 7 marzo de 1997. Min. Obras Públicas. D.G.A. 1997.

* Caudal Promedio río Lluta entre 28 febrero y 3 marzo de 1997. Min. Obras Públicas. D.G.A. 1997.

** Caudal Promedio mensual. Min. Obras Públicas. D.G.A. 2006.

Cabe mencionar que el caudal promedio mensual para Lluta, estación Tocontasi del año 2004 está incompleto, porque el registro observado en la página web de la Dirección General de Aguas (D.G.A.) está controlado sólo entre el 1 y 12 de

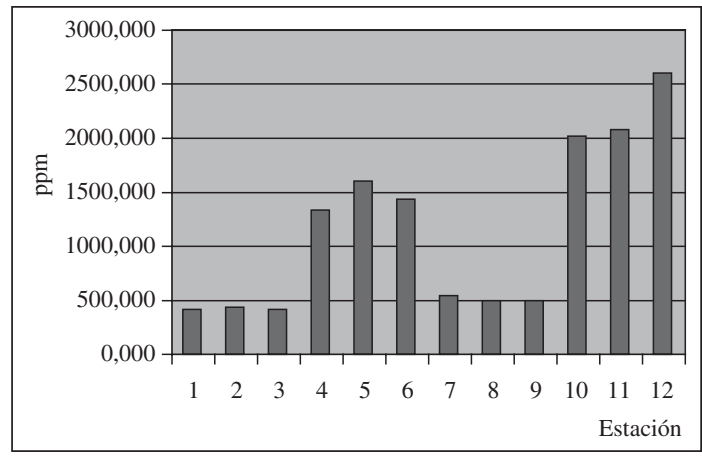

Gráfico 1. Sólidos Totales por Estación y año. febrero del 2004 y el muestreo fue hecho el 17 de febrero de ese año. Igual ocurre con Lluta en la estación Alcérreca año 2005, cuyo registro es sólo entre el 1 y 6 de febrero. El muestreo se hizo el 15 de febrero de ese año.

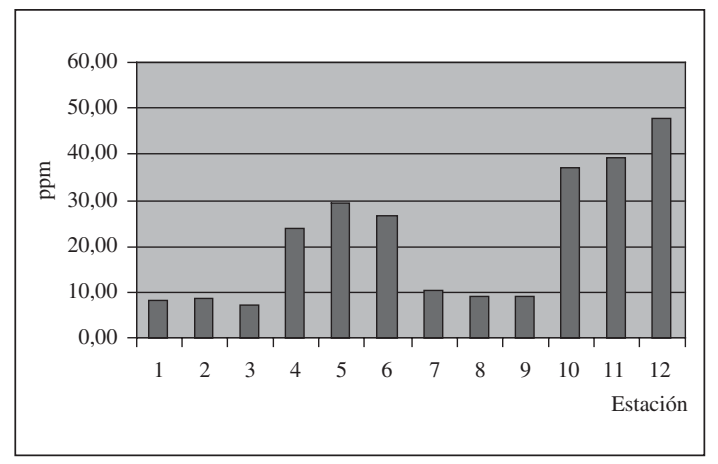

Gráfico 2. Sólidos Disueltos por Estación y año. 
Tabla 3

Sólidos totales, disueltos y suspendidos por estación

\begin{tabular}{|c|c|c|c|c|c|}
\hline ESTACIÓN & Muestra $\mathbf{N}^{\circ}$ & Fecha & $\begin{array}{c}\text { SS.Totales } \\
\mathrm{mg} / \mathrm{L}\end{array}$ & $\begin{array}{c}\text { SS.Disueltos } \\
\mathrm{mg} / \mathrm{L}\end{array}$ & $\begin{array}{c}\text { SS.Suspendidos } \\
\mathrm{mg} / \mathrm{L}\end{array}$ \\
\hline Azapa, Saucache & 1 & 4 a 7/03/1997 & 620,0 & N. D. & 30,7 \\
\hline Azapa, Puente FFCC & 2 & $"$ & 220,0 & N. D. & 36,0 \\
\hline Azapa, Desembocadura & 3 & $"$ & $16.860,0$ & N. D. & 13,3 \\
\hline Lluta, Sta. Lucía & 4 & $28 / 02$ a $3 / 03 / 1997$ & 800,0 & N. D. & 19,0 \\
\hline Lluta, Panamericana & 5 & $"$ & 940,0 & N. D. & 17,3 \\
\hline Lluta, Desembocadura & 6 & $"$ & $4.240,0$ & N. D. & 28,0 \\
\hline Azapa, Las Maitas & 7 & $02 / 2000$ & $6.135,0$ & 0,300 & N. D. \\
\hline Azapa, Saucache & 8 & $"$ & $7.605,0$ & 0,330 & N. D. \\
\hline Azapa, Puente FF CC & 9 & $"$ & $8.230,0$ & 0,2950 & N. D. \\
\hline Azapa, Desembocadura & 10 & $"$ & $9.140,0$ & 0,295 & N. D. \\
\hline Azapa, Las Maitas & 11 & $13 / 02 / 2001$ & 10,54 & N. D. & N. D. \\
\hline Azapa, Saucache & 12 & $"$ & 10,04 & N. D. & N. D. \\
\hline Azapa, Puente Ferroc. & 13 & $"$ & 13,38 & N. D. & N. D. \\
\hline Azapa, Desembocadura & 14 & $"$ & $11.700,0$ & N. D. & N. D. \\
\hline Lluta, Desembocadura & 15 & & 30.660 & N. D. & N. D. \\
\hline Azapa, Las Maitas & 16 & $17 / 02 / 2004$ & 420.000 & 8,20 & 411,8 \\
\hline Azapa, Saucache & 17 & $"$ & 430,000 & 8,40 & 421,6 \\
\hline Azapa, Desembocadura & 18 & $"$ & 410,000 & 7,20 & 402,8 \\
\hline Lluta, Santa Lucía & 19 & $17 / 02 / 2004$ & $1.335,0$ & 24,10 & $1.310,9$ \\
\hline Lluta, Panamericana & 20 & $"$ & $1.430,0$ & 26,60 & $1.403,4$ \\
\hline Lluta, Desembocadura & 21 & $"$ & $1.600,0$ & 29,10 & $1.570,9$ \\
\hline Azapa, Las Maitas & 22 & $15 / 02 / 2005$ & 535,000 & 10,40 & 524,600 \\
\hline Azapa, Saucache & 23 & $"$ & 495,000 & 9,10 & 485,900 \\
\hline Azapa, Desembocadura & 24 & $"$ & 500,000 & 9,10 & 490,900 \\
\hline Lluta, Santa Lucía & 25 & $15 / 02 / 2005$ & $2.025,000$ & 36,80 & $1.988,200$ \\
\hline Lluta, Panamericana & 26 & $"$ & $2.085,000$ & 39,10 & $2.045,900$ \\
\hline Lluta, Desembocadura & 27 & $"$ & $2.595,000$ & 48,00 & $2.547,000$ \\
\hline
\end{tabular}

ND: No determinado.

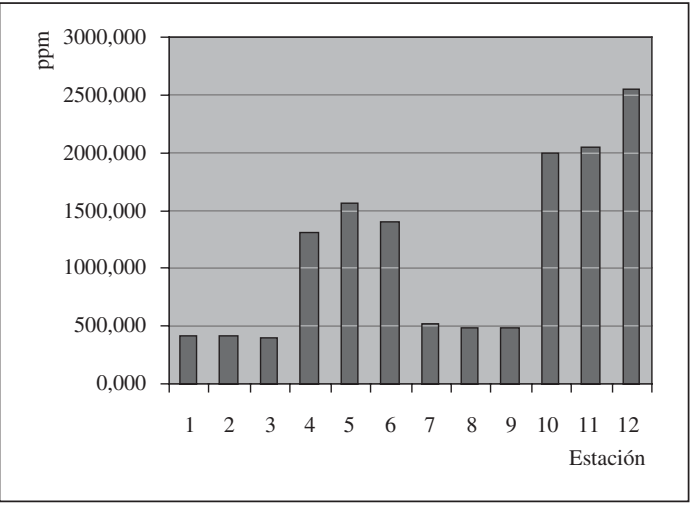

Gráfico 3. Sólidos Suspendidos y Sedimentables por Estación y año. 
Tabla 4

Aporte teórico total estimado de material particulado suspendido y sedimentable en función del tiempo considerando sólo el día del muestreo en ambos ríos

\begin{tabular}{|c|c|c|c|c|c|}
\hline Fecha & Estación & $\begin{array}{c}\text { Caudal Medio } \\
\text { en } \mathrm{m}^{3} / \mathrm{seg}\end{array}$ & $\begin{array}{c}\text { Aporte en } \\
\text { m³}^{3} / \text { hora }\end{array}$ & $\begin{array}{c}\text { Caudal en } \\
\text { m³/día }^{3}\end{array}$ & $\begin{array}{c}\text { Toneladas } \\
\text { de aporte día }\end{array}$ \\
\hline $17-02-2004$ & Azapa, Las Maitas & 1,6 & 5.760 & 138.240 & 56,93 \\
\hline " & Azapa, Saucache & 1,6 & 5.760 & 138.240 & 58,28 \\
\hline$"$ & Azapa, Desembocadura & 1,6 & 5.760 & 138.240 & 55,68 \\
\hline " & Lluta, Panamericana & 3,1 & 11.160 & 267.840 & 375,89 \\
\hline " & Lluta, Desembocadura & 3,1 & 11.160 & 267.840 & 420,75 \\
\hline $15-02-2005$ & Azapa, Las Maitas & 1,75 & 6.300 & 151.200 & 79,32 \\
\hline$"$ & Azapa, Saucache & 1,75 & 6.300 & 151.200 & 73,47 \\
\hline$"$ & Lluta, Panamericana & 7,75 & 27.900 & 669.600 & $1.369,93$ \\
\hline$"$ & Lluta, Desembocadura & 7,75 & 27.900 & 669.600 & $1.705,47$ \\
\hline
\end{tabular}

\section{DISCUSIÓN}

En términos generales, en la Tabla 2 y los gráficos 1,2 y 3 se observa que durante los mismos eventos de bajada o aluviones de los años 2004 y 2005, registrados en este trabajo, el río Lluta transporta mayor cantidad de sólidos totales, disueltos y suspendidos sedimentables que el río San José, lo cual está asociado al mayor caudal registrado en dicha cuenca (Horne y Goldman, 1994), como tendencia general observada en la Tabla 3.

Cabe mencionar que la estación Alcérreca, por estar ubicada en la zona climática desértico marginal de altura (Köeppen en Bol. CONAF 1978), recibe menos aportes de otros cursos de agua explicando la diferencia de caudal con la estación Tocontasi, durante los periodos de muestreo y de acuerdo con los registros observados en la página web de la Dirección General de Aguas (D. G. A.) dependiente del Ministerio de Obras Públicas (M. O. P. 2004).

En la Tabla 4 se observan los aportes de material particulado suspendido y sedimentable, estimado sólo para el día de muestreo, por lo que el aporte que hacen ambos ríos en la zona costera de la rada de Arica de material suspendido y sedimentable a la zona costera debe ser mayor si se considera que este tipo de evento climático dura varios días. Cabe mencionar que de acuerdo con Senior (1987) la fuerza iónica del agua de mar contribuye a aglomerar las partículas suspendidas y dispersas en el agua fluvial, aumentando su peso específico haciéndolas sedimentar y depositar, en este caso, en el fondo de la zona costera de la rada de Arica. Esto ocurre principalmente como resultado de la formación de un filtro geológico natural en la zona transicional entre la masa de agua salada oceánica y la dulce continental, fenómeno que ocurre alrededor del $10 \%$ de salinidad. Esta condición eventualmente se puede generar en la desembocadura del río San José, por su condición de aguas calmas o más tranquilas resultado de su cercanía al molo de abrigo, mientras que en la desembocadura del río Lluta, por la persistencia e intensidad del tren de olas que se registra de forma normal, es más difícil la formación de una zona transicional continua desde el río al mar, por lo que la dispersión del material particulado de origen continental y transporte fluvial se produce a lo largo de la costa, dispersándose estos aglomerados mar adentro.

Estos eventos tienen marcados efectos en la ciudad de Arica y su temporada turística, la que concluye abruptamente cuando se producen estos eventos fluviales que alteran las características normales de las aguas y sectores recreacionales 
costeros, al modificarse el color del agua en los balnearios de los sectores Chinchorro (Figura 14) y Las Machas. Por esta razón se produce la no concurrencia de los turistas a estos balnearios retirándose inclusive de la ciudad, razón por la cual se les atribuye un efecto económico negativo a estos eventos, los que, en opinión de muchos, perjudican la temporada turística de la ciudad.

Las Figuras 3, 4, 5, 7, 8, 9, 10 y 11 permiten comparar el aspecto visual del caudal en sectores de las diferentes estaciones durante el período aluvional y sin éste, como se observa en las Figuras 5-6 y 11-12 para los sectores Puente ferroviario del río San José y Puente vial del río Lluta, respectivamente.

\section{CONCLUSIÓN}

De lo anterior se puede concluir que durante las "bajadas" o eventos aluvionales de los ríos Lluta y San José hacia la rada de Arica se producen interesantes aportes de material particulado sedimentable, incrementando los volúmenes de sedimentos costeros marinos cercanos a ambas desembocaduras.

Los aportes sedimentarios fluviales y la hidrodinámica del sector del río San José se ven influidos por la dársena portuaria, incrementando el embanque de la ribera sur de la desembocadura

\section{LITERATURA CITADA}

APHA, AWWA, WPCF, 1992. Métodos normalizados para el análisis de aguas potables y residuales. Ediciones Díaz de Santos, S. A.

PLAN PARA EL MANEJO DEL PARQUE NACIONAL LAUCA, 1978. Boletín CONAF. Arica.

CAMPOS ROJAS, GISELLE. 2005. Localización de un Embalse en el río San José y su Impacto Económico. Informe Ingeniería Civil Industrial. Universidad de Tarapacá.

JAPAN INTERNATIONAL COOPERATION AGENCY (JICA), MARCH 1995. The Study on the Development of Water Resources in Northern Chile. Main Report, y modificando inclusive la geomorfología del sector al originar una península con los aportes de este material particulado de origen continental, transportado fluvialmente.

Los aportes de sólidos sedimentables que se producen en ambas cuencas corresponden a un proceso erosivo de tipo natural, sin origen ni intervención antrópica, respondiendo a eventos climáticos de ocurrencia casi regular, por lo que se pueden esperar importantes aportes de sólidos sedimentables durante estos eventos estivales. La cantidad de material aportado se sugiere sea considerada ante cualquier proceso de toma de decisión de construcción de alguna obra regulatoria de caudal en cualquiera de las cuencas objeto de estudio, las cuales deben de considerar el aporte de sólidos que afectarán de manera dramática los volúmenes de diseño de las mismas.

Por ser el proceso de erosión, arrastre, colmatación y sedimentación de sólidos suspendidos un proceso dinámico que afecta al cauce principal en todo su recorrido, trayecto que se ve alterado por la destrucción de estructuras de suelos y obras públicas resultado del paso del torrente, se considera oportuno desarrollar trabajos de control de caudales en la parte alta de ambas cuencas, esfuerzo que permitiría asegurar un control eficiente del aporte de materia sedimentable en los cursos de los ríos Lluta y San José de la Provincia de Arica.
Supporting Report A: Surface Water. Pacific Consultants International. Tokyo. Japan.

DIRECCIÓN GENERAL DE AGUAS (D.G.A.), 2004. Diagnóstico y Clasificación de los Cursos y Cuerpos de Agua Según Objetivos de Calidad. Cuenca del río Lluta.

HORNE, A. J. \& C. R. GOLDMAN. 1994. Limnology. McGraw-Hill, Inc.

INN. 1994. Norma Chilena $N^{\circ}$ 411/6. Of. 98.

SENIOR, WILLIAM. 1987. Guía práctica de Análisis Químico del Agua de Mar. Instituto Oceanográfico. Núcleo de Sucre, Universidad de Oriente. Venezuela.

WETZEL, R. G. 1975. Limnology. W. B. Saunders Company. 